\title{
Pristimerin Inhibits Adjuvant Arthritis Fibroblast Like Synoviocytes Cell Proliferation through Cell Cycle Arrest and Induction of Apoptosisa
}

\author{
SHUTONG BAI ${ }^{1}$, WANJIAO GAO, QIUDI DENG, XIAOCHUN LIN, J. ZHENG, YUYAO CHEN AND LI TONG*
}

School of Chinese Medicine, Southern Medical University, Guangzhou 510515,GuangDong, China, ${ }^{1}$ Chongqing Hospital of Traditional Chinese Medicine, Chongqing Key Laboratory of Traditional Chinese Medicine to Prevent and Treat Autoimmune Diseases, Chongqing 400021, China

Bai et al.: Pristimerin inhibits AA FLS cell proliferation

To investigate the effect of pristimerin, an effective component of maple leaf, on the cell cycle and apoptosis of adjuvant arthritis fibroblast like synoviocytes adjuvant arthritis fibroblast like synoviocytes cells. Adjuvant arthritis fibroblast like synoviocytes cells were cultured in vitro, the effect of pristimerin on the proliferation of adjuvant arthritis fibroblast like synoviocytes cells was analyzed by 3-(4,5-dimethylthiazol2-yl)-2,5-diphenyl tetrazolium bromide method, and the effect of pristimerin on adjuvant arthritis fibroblast like synoviocytes cell cycle was analyzed by flow cytometry. Hoechst 33258 staining experiment and flow cytometry were used to detect the effect of pristimerin on cell apoptosis of adjuvant arthritis fibroblast like synoviocytes cells. The effect of pristimerin on mitochondrial membrane of adjuvant arthritis fibroblast like synoviocytes cells was analyzed by flow cytometry. Western Blotting was used to detect the effect of pristimerin on the expression of adjuvant arthritis fibroblast like synoviocytes mitochondrial pathway regulators Bax, B-cell lymphoma 2 and Caspase-3. The results of 3-(4,5-dimethylthiazol-2-yl)-2,5diphenyl tetrazolium bromide experiments showed that pristimerin had a significant inhibitory effect on the proliferation of adjuvant arthritis fibroblast like synoviocytes cells in a dose-dependent manner. The half inhibitory concentration of pristimerin on adjuvant arthritis fibroblast like synoviocytes cells was $2.97 \mu \mathrm{M}$. Cell cycle results showed that $1.5 \mu \mathrm{M}$ and $3 \mu \mathrm{M}$ pristimerin could significantly increase the number of cells in the Gap 0/Gap 1 phase $(p<0.05)$ and decrease the number of cells in the synthesis phase $(p<0.01)$. Hoechst 33258 staining experiment and flow cytometry detection of apoptosis showed that the rate of early apoptotic cells in adjuvant arthritis fibroblast like synoviocyteswas $17.307 \pm 2.519 \%$ in the $0.75 \mu \mathrm{M}$ scopolamine group, which was not statistically different from the control group $(\mathrm{p}>0.05)$. The apoptosis rate of adjuvant arthritis fibroblast like synoviocytes cells in the $1.5 \mu \mathrm{M}$ group was $47.413 \pm 2.503 \%$, and the apoptosis rate of adjuvant arthritis fibroblast like synoviocytes cells in the $3 \mu \mathrm{M}$ group was $\mathbf{7 0 . 9 7 0}$ $\pm 4.205 \%$, which were significantly higher than those in the control group $(p<0.01)$. Cell membrane potential test results showed that the proportion of adjuvant arthritis fibroblast like synoviocytes depolarized cells in the pristimerin $1.5 \mu \mathrm{M}$ group and the $3 \mu \mathrm{M}$ group were $54.550 \pm 3.056 \%$ and $91.373 \pm 3.163 \%$, which were significantly higher than the control cell depolarization rate of $38.480 \pm 7.447 \%(p<0.01)$, pristimerin $0.75 \mu \mathrm{M}$ had no significant effect on adjuvant arthritis fibroblast like synoviocytes mitochondrial membrane potential $(p>0.05)$. Western Blotting results showed that compared with normal fibroblast like synoviocytes cells, the relative protein levels of B-cell lymphoma 2 , Pro-Caspase-3 and Cleave-Caspase- 3 in adjuvant arthritis fibroblast like synoviocytes cells were significantly increased $(p<0.01)$, while the expression of Bax in adjuvant arthritis fibroblast like synoviocytes cells was decreased but not statistically different $(p>0.05)$. The relative expression of B-cell lymphoma 2 , Pro-Caspase-3 and Cleave-Caspase-3 in pristimerin-1 $\mu \mathrm{M}$ and $3 \mu \mathrm{M}$ groups was significantly lower than that of adjuvant arthritis fibroblast like synoviocytes group $(p<0.01)$, while the expression of Bax was higher than that of adjuvant arthritis fibroblast like synoviocytes group $(\mathbf{p}<\mathbf{0 . 0 5})$. Pristimerin can induce adjuvant arthritis fibroblast like synoviocytes apoptosis through the cell cycle arrest and regulate the mitochondrial apoptotic pathway to inhibit the adjuvant arthritis fibroblast like synoviocytes cell hyper proliferation and achieve Anti-rheumatoid arthritis synovial hyperplasiay.

Key words: Rheumatoid arthritis, pristimerin, maple, fibroblast like synoviocyte, apoptosis, cell cycle 
Rheumatoid arthritis (RA) is an autoimmune system disease with chronic, recurrent arthritis as the main clinical symbol, with a worldwide incidence of approximately $1 \%$. Due to its long course and high disability, it has also become an unread cancer, placing a heavy burden on patients and society ${ }^{[1]}$. The typical pathological features of RA are abnormal proliferation of synovial tissue, infiltration of inflammatory cells, formation of blood vessels and erosion of bone. Studies have shown that fibroblast like synoviocytes (FLS) are the key effector cells that cause RA synovial lining lesions and joint damage ${ }^{[2]}$. The normal human synovial tissue lining layer is composed of 2 to 4 layers of FLS cells, while RA FLS shows signs of abnormal activation early in the onset of RA, showing similar proliferation and anti-apoptotic characteristics similar to tumour cells, making the number of layers in FLS cells reaches 10 to 20 layers $^{[3]}$. A large number of excessively proliferating RA FLS directly cause the proliferation of synovial tissues. At the same time, RA FLS cells secrete pro-inflammatory cytokines, growth factors and matrix metalloproteinase in the joint cavity microenvironment and interact with macrophages, monocytes, $\mathrm{T}$ lymphocytes, and $\mathrm{B}$ lymphocytes to continuously amplify inflammation signals in the joint cavity, promoting the formation of vascular crests, and continuously increasing joint damage. Therefore, suppressing the excessive capital increase of FLS is of great significance in delaying the condition of RA.

Studies have shown that compared with FLS cells of patients with osteoarthritis, FLS cells of RA patients have significant anti-apoptotic properties and cell cycle changes, with prominent manifestations of vigorous cell division and anti-apoptosis ${ }^{[4]}$. Studies have shown that the enhanced anti-apoptotic ability of FLS in RA patients is closely related to the abnormal expression of anti-apoptotic protein of B-cell lymphoma-2 (Bcl-2). Compared withnormalFLS, there is a significant increase in Bcl-2 expression and a decrease in Bax expression in RA FLS cells. As an important apoptosis-regulating protein, Bcl-2 regulates mitochondrial membrane permeability by binding to Bax protein. Control the opening and closing of mitochondrial membrane pathways to maintain membrane potential stability ${ }^{[5]}$. Under normal conditions, Bax protein is located in the cytoplasm as a monomer, and a few are presented in organelles such as the endoplasmic reticulum. Bcl-2 protein is distributed on the mitochondrial membrane,

*Address for correspondence E-mail: duojiang8818171470@163.com endoplasmic reticulum membrane, and outer nuclear membrane. After cells receive the death message, Bax protein was quickly inserted into the mitochondrial membrane. After combining with the voltage-dependent anion channel and adenine nucleotide transferase on the mitochondrial membrane, the mitochondrial membrane channel was opened to regulate the release of cytochrome $\mathrm{C}$ in the mitochondrial membrane and cut Pro-Caspase- 3 in the cytoplasm; and CleaveCaspase-3, which is activated after splicing, can further cleave the substrate Poly(ADP-ribose) polymerase (PARP), thereby completing the apoptosis process ${ }^{[6,7]}$. However, in RA FLS cells, due to the expression of Bcl-2 protein was significantly increased, it caused the balance of mitochondrial apoptotic pathway to be broken, resulting in the inability of cells to complete metabolism through the apoptotic program, which is also one of the important reasons leading to the excessive proliferation of RA FLS.

Pristimerin (PM) is the main active triterpene component of the Chinese herbal medicine (Celastrus aculeatus Merr), which is from the ethyl acetate extraction from ethanol ${ }^{[8]}$, with molecular formula $\mathrm{C}_{30} \mathrm{H}_{42} \mathrm{O}_{4}$ and molecular weight 464.46. Previous studies have shown that this component has reduced Adjuvant arthritis (AA) rat joint swelling and arthritic scoring effect. At the same time, this component can inhibit joint pathological changes and inhibit proinflammatory factor secretion in AA rats, and has significant anti-RA activity ${ }^{[9]}$. However, PM's anti-RA effect through cooperative drug action mechanisms and pathways remains to be revealed by further research. This study used AA FLS cells cultured in vitro as the research object, and analyzed the effect of PM on the proliferation and apoptotic process of AA FLS cells. The research showed that PM can inhibit AA FLS proliferation through cell cycle arrest and was dose-dependent. At the same time, PM can affect the mitochondrial apoptosis pathway by regulating the expression ratio of $\mathrm{BAX} / \mathrm{Bcl}-2$ protein, promote the depolarization of mitochondrial membrane potential of AA FLS cells, shear and activate Caspase 3, induce AA FLS apoptosis, and thus inhibit AA FLS cell hyper

\footnotetext{
This is an open access article distributed under the terms of the Creative Commons Attribution-NonCommercial-ShareAlike 3.0 License, which allows others to remix, tweak, and build upon the work non-commercially, as long as the author is credited and the new creations are licensed under the identical terms
}

Accepted 14 December 2020

Revised 04 December 2020 Received 11 April 2020 Indian J Pharm Sci 2020;82(5):819-827 
proliferation. This article explores the pharmacological action mechanism of PM against RA synovial hyperplasia from the perspective of inhibiting cell proliferation and inducing apoptosis, and it is the basis of accumulated experimental data for the development of new drugs based on PM.

\section{MATERIALS AND METHODS}

\section{Experimental animals:}

SPF Sprague Dawley rats (SD rats), male, 5-6 w of age, body weight $130 \pm 10 \mathrm{~g}$, purchased from the Experimental Animal Center of Southern Medical University, animal certificate number:SCXK (Guangdong) 2011-0015. Rats were housed in an Specific Pathogen Free (SPF) laboratory (Laboratory Animal Center of Southern Medical University), room temperature $25 \pm 1^{\circ}$, humidity 50-70 \% and free drinking and eating.

\section{Drugs and reagents:}

Oblate (Guangzhou Brand Biological Products Co., Ltd., China); Dulbecco's Modified Eagle's medium (DMEM) low sugar medium (Hyclone, USA); heatkilled conjugated Mycobacterium (Mycobacterium tuberculosis (Mtb), Defico laboratory, USA); premium Fetal Bovine Serum (Hangzhou Sijiqing Biological Company, China); Type II Collagenase (MP Company, USA); Trypsin (Gibco Company, USA); Mineral Oil (Sigma Company, USA); Penicillin and Streptomycin (Gino Bio Products Co., Ltd.); Bax, $\beta$-actin polyclonal primary antibody (Shanghai Biological Engineering Co., Ltd., China); Bcl-2, Caspase-3 polyclonal primary antibody, goat anti-rabbit IgG (Cell Signaling, USA).

\section{AA rat model preparation:}

The AA rat preparation method is consistent with that described in the previous paper ${ }^{[10]}$. Mtb powder was fully ground under aseptic conditions, and then an appropriate amount of mineral oil was added, thoroughly mix Mtb with the mineral oil, and then an appropriate amount of mineral oil was added to make the final concentration of the Mtb suspension to $7.5 \mathrm{mg} /$ $\mathrm{mL}$. The rats were fixed, and $0.1 \mathrm{~mL}(1.5 \mathrm{mg} /$ head $)$ of Mtb suspension was injected subcutaneously at the root of the tail and the left hind foot, respectively. The alcohol cotton ball was pressed against the injection point to prevent leakage.

\section{Primary culture of AA FLS cells:}

AA rats with successful modelling were sacrificed by cervical dislocation. After soaking in $75 \%$ ethanol for
15 min, bilateral synovial tissues of knee joints were washed twice with Phosphate-buffered saline (PBS) solution, cut into $1 \mathrm{~mm}^{3}$ tissue pieces, and added with $0.4 \%$ concentration of type II collagenase for digestion and cultured in a $5 \% \mathrm{CO}_{2}$ cell incubator at $37^{\circ}$ for $4 \mathrm{~h}$. Centrifuge the tissue digestion solution $(2500 \mathrm{rpm} / \mathrm{min}$, $5 \mathrm{~min})$ to remove the supernatant. Add the complete culture medium (10\% fetal bovine serum+DMEM low sugar medium+penicillin+streptomycin) to $37^{\circ}, 5 \%$ $\mathrm{CO}_{2}$ cell incubator overnight. Change the solution the next day to remove non adherent cells. FLS cells were cultured in complete medium, and the medium was changed once every $48 \mathrm{~h}$. AA FLS cells of the 3 rd to 6th generation were used for experiments ${ }^{[11]}$.

\section{3-(4,5-dimethylthiazol-2-yl)-2,5-diphenyl tetrazolium bromide (MTT) experiment:}

AA FLS cells were seeded into a 96-well plate $(5 \times 103$ cells/well $)$ and placed in a $5 \% \mathrm{CO}_{2}$ cell incubator overnight to adhere the cells. Analysis PM $(0.2,0.4,0.8,1.5,3$, and $6 \mu \mathrm{mol} / \mathrm{L})$ with different concentrations was co-cultured with AA FLS cells, and a complete medium containing $0.01 \%$ DMSO was used as a negative control, with 5 replicates in each group. After $24 \mathrm{~h}$ of drug intervention, add $16 \mu \mathrm{L}$ of MTT solution $(5 \mathrm{mg} / \mathrm{mL})$ to each well, place the culture plate in the cell incubator for $4 \mathrm{~h}$, discard the supernatant, add $150 \mu \mathrm{L}$ of DMSO solution, and shake for $10 \mathrm{~min}$ to fully lysis. Microplate reader (wavelength $620 \mathrm{~nm}$ ) was used to detect the absorbance of each well, calculate the effect of scopolamine on the viability of AA FLS cells and the $24 \mathrm{~h}$ half inhibitory concentration (IC50).

\section{Detection of AA-FLS cell cycle by flow cytometry:}

AA FLS was inoculated into 6-well plates at a density of $5 \times 106$ cells/well, placed in a $37^{\circ}, 5 \% \mathrm{CO}_{2}$ cell incubator overnight, and replaced with serum-free DMEM medium the next day for $24 \mathrm{~h}$. AA FLS was treated with $\mathrm{PM}$ of $3 \mu \mathrm{M}, 1.5 \mu \mathrm{M}$, and $0.75 \mu \mathrm{M}$ for $24 \mathrm{~h}$, and a complete medium containing $0.01 \%$ DMSO was used as a negative control. Cells were collected after $24 \mathrm{~h}$ (floating cells in the medium at $800 \mathrm{rpm} /$ min, and centrifuged for $5 \mathrm{~min}$ to collect). Adherent cells were trypsinized and collected by centrifugation. Cells were washed once with PBS and adjusted to a cell concentration of $1 \times 106$ cells $/ \mathrm{mL}$. Take $1 \mathrm{~mL}$ of cell suspension, discard the supernatant after centrifugation, add $500 \mu \mathrm{L}$ of $70 \%$ ethanol, and fix at $4^{\circ}$ in the dark for $2 \mathrm{~h}$. Add $100 \mu \mathrm{L}$ of RNase in a $37^{\circ}$ water bath for $30 \mathrm{~min}$, wash once in PBS, add $400 \mu \mathrm{L}$ of propidium 
iodide (PI) at $4^{\circ}$ in the dark for $30 \mathrm{~min}$, and then detect by flow cytometry.

\section{Hoechst 33258 staining:}

The cell processing method was the same as described in above. After $24 \mathrm{~h}$ of AA FLS PM intervention, cells were collected from each group, washed twice in PBS, fixed in a $4 \%$ formaldehyde solution at $4^{\circ}$ for $10 \mathrm{~min}$, and washed twice in PBS to adjust the cell concentration to $1 \times 105$ cells $/ \mathrm{mL}$, take $50 \mu \mathrm{L}$ of cell suspension smear, dry naturally, add $50 \mu \mathrm{L}$ of Hoechst 33258 dye, and stain at room temperature for $15 \mathrm{~min}$ in the dark, wash the slides to remove excess water, observe the cell morphology under a UV microscope and take a picture recording.

\section{Detection of Apoptosis by Flow Cytometry:}

Cell processing method is the same as described above. Flow cytometry was performed according to the instructions of Annexin V-FITC/7-Amino-Actinomycin (7AAD) Apoptosis Detection Kit. After $24 \mathrm{~h}$ of PM intervention with AA FLS, cells were collected, washed with pre-chilled PBS twice, and the cell concentration was adjusted to $1 \times 106$ cells $/ \mathrm{mL}$ with $1 \times$ binding buffer. $100 \mu \mathrm{L}$ of the cell suspension was added to the Falcon tube, and $5 \mu \mathrm{L}$ of Annexin was added respectively. V-FITC and 7 AAD dyes were mixed and stained at room temperature for $15 \mathrm{~min}$ in the dark, and then $400 \mu \mathrm{L}$ of $1 \times$ binding buffer was added for detection by flow cytometry.

\section{Detection of mitochondrial membrane potential by flow cytometry:}

The JC-1 staining method was used, and the experimental operation was performed according to the instructions of the JC-1 mitochondrial membrane potential detection kit (KGI). After $12 \mathrm{~h}$ of AA FLS intervention with $3 \mu \mathrm{M}, 1.5 \mu \mathrm{M}$, and $0.75 \mu \mathrm{M} \mathrm{PM}$, cells were collected, washed twice with PBS, and the cell concentration was adjusted to $5 \times 10^{5}$ cells $/ \mathrm{mL}$ with $1 \times$ binding buffer. $100 \mu \mathrm{L}$ of the cell suspension was added to Falcon Tube, and then add $5 \mu \mathrm{L}$ of JC- 1 dye and mix. After staining at $37^{\circ}$ for $30 \mathrm{~min}$, add $400 \mu \mathrm{L}$ of $1 \times$ binding buffer, and measure the mitochondrial membrane potential by flow cytometry.

\section{Western Blotting Experiment:}

The cell processing method is the same as described above. Cells were collected by centrifugation of AA FLS after PM intervention for $24 \mathrm{~h}$. Cells were lysed on ice to extract total protein and quantify. $40 \mu \mathrm{g}$ of total protein extract was separated by sodium dodecyl sulfate polyacrylamide gel electrophoresis (SDS-PAGE). The membrane was transferred to $250 \mathrm{~mA}$ for $90 \mathrm{~min}$. The Polyvinylidene fluoride (PVDF) membrane was blocked with $5 \%$ skimmed milk powder for $2 \mathrm{~h}$ at room temperature. The membrane was washed 3 times with Tris-buffered saline with $0.1 \%$ Polysorbate 20 (TBS-T) for 5 min each. $\beta$-actin, Bax, Bcl-2, and Caspase- 3 were diluted at a ratio of $1: 1000$. The PVDF membrane was placed in the corresponding protein primary antibody solution and incubated overnight at $4^{\circ}$. The membrane was washed 3 times with TBS-T for $5 \mathrm{~min}$. The root peroxidase-labelled secondary antibody was incubated at room temperature for $2 \mathrm{~h}$, developed by ECL, and the target band was detected by machine exposure (Kodak, USA). Image J (1.48 NIH, USA) software measures the gray value of the target band.

\section{Statistical analysis:}

SPSS 19.0 software was used to statistically analyse the experimental results. The experimental data were tested for variance homogeneity and normality. The measurement data were analyzed by One-way Analysis of variance (ANOVA) method. The data were expressed as mean \pm standard deviation $(\mathrm{x} \pm \mathrm{s})$. Statistics Results were considered statistically significant at $\mathrm{p}<0.05$.

\section{RESULTS AND DISCUSSION}

Cells proliferate slowly after passage for 1 to $3 \mathrm{~d}$, and the growth curves of both cells show a gentle upward trend; the proliferation of cells accelerates on the 4th to 7 th $\mathrm{d}$. The speed of proliferation of AA-FLS begins on the 4th $d$ was significantly higher than that of FLS cells in normal rats, and there was a statistical difference $(\mathrm{p}<0.05)$ (fig. 1).

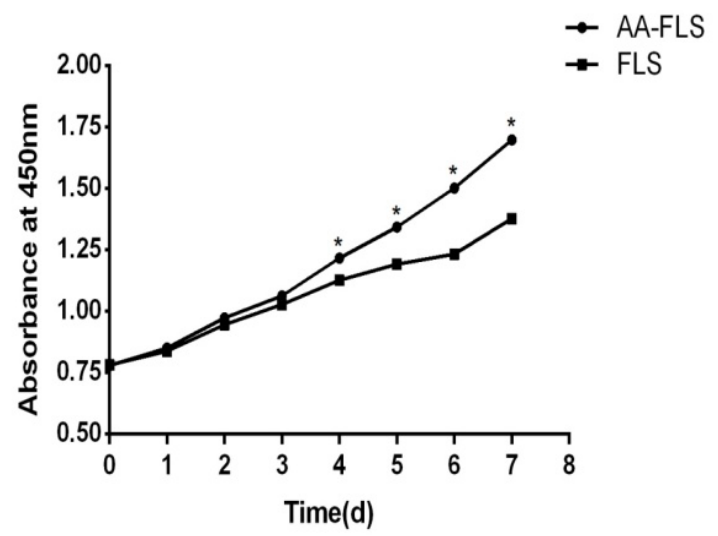

Fig. 1: The proliferation curve of FLSs

*p $<0.05$ vs. FLS group 
The results of MTT experiments showed that with the increase of the dose of PM, the 24-h survival rate of AA FLS cells showed a downward trend and was dosedependent. When the PM dose was $0.4 \mu \mathrm{M}$, the survival rate of AA FLS cells was not statistically different from that of the control group. When the dose was increased to $0.8 \mu \mathrm{M}$, the AA FLS survival rate began to decrease significantly. When the PM dose reached $1.5 \mu \mathrm{M}$, the AA FLS survival rate was further reduced, compared with the control group. The experimental results showed that scopolamine could inhibit the activity of AA FLS, and the 24-h IC50 of PM against AA FLS was $2.97 \mu \mathrm{M}$ (fig. 2 and fig. 3).

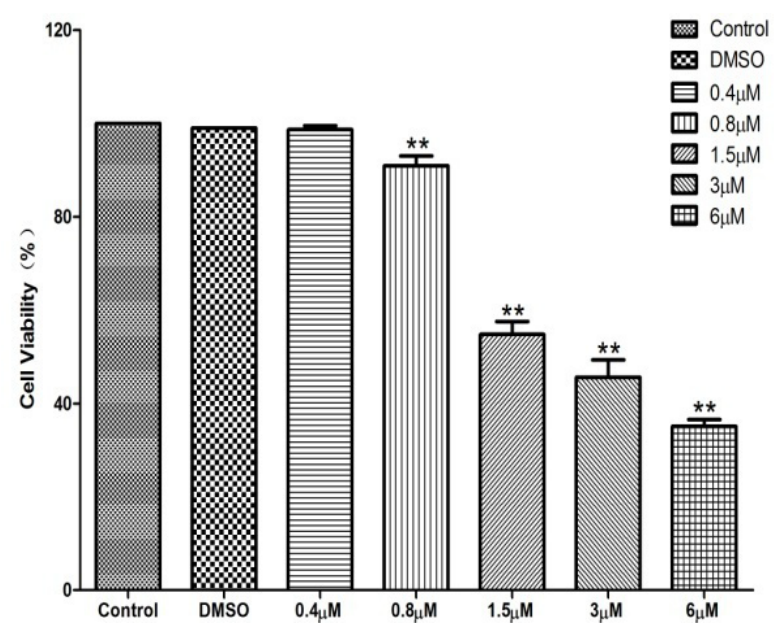

Fig. 2: Effect of PM on cell proliferation in AA FLS ( $(\overline{\mathbf{x}} \pm \mathbf{s})$ $* * \mathbf{p}<0.01$ vs. control group
Flow cytometry was used to detect the effect of PM on the AA-FLS cell cycle, and the results were found (see fig. 4) PM high, medium, and low dose groups increased the number of Gap 0/Gap 1 (G0/G1) cells, of which the difference between the PM $0.75 \mu \mathrm{M}$ group and the control group was not statistically significant, and the difference between the $1.5 \mu \mathrm{M}$ group and the $3 \mu \mathrm{M}$ group was statistically significant compared with the control group $(p<0.05)$; the number of S-phase cells decreased after PM intervention. The difference between the high-, medium-, and low-dose group and the control group was statistically significant $(\mathrm{p}<0.01)$. The experimental results show that PM can interfere with the AA-FLS cell cycle, block cells in the G0/G1 phase, and the blocking effect is dose-dependent.

Hoechst 33258 experimental results found that: in the negative control group, the nucleus mostly showed diffuse light blue fluorescence, and a small number of cells showed bright blue fluorescence. Apoptosis rate of AA FLS cells in the low, medium, and high dose groups of PM was gradually increased with the increase in the dose. The three groups were statistically different from the control group. Flow cytometry was used to detect PM on AA FLS. The effect of apoptosis, the experimental results found that: the negative control cells were concentrated in the lower left limit (live cells), a small number of cells were in the lower right limit (early apoptotic cells) and right upper limit (late

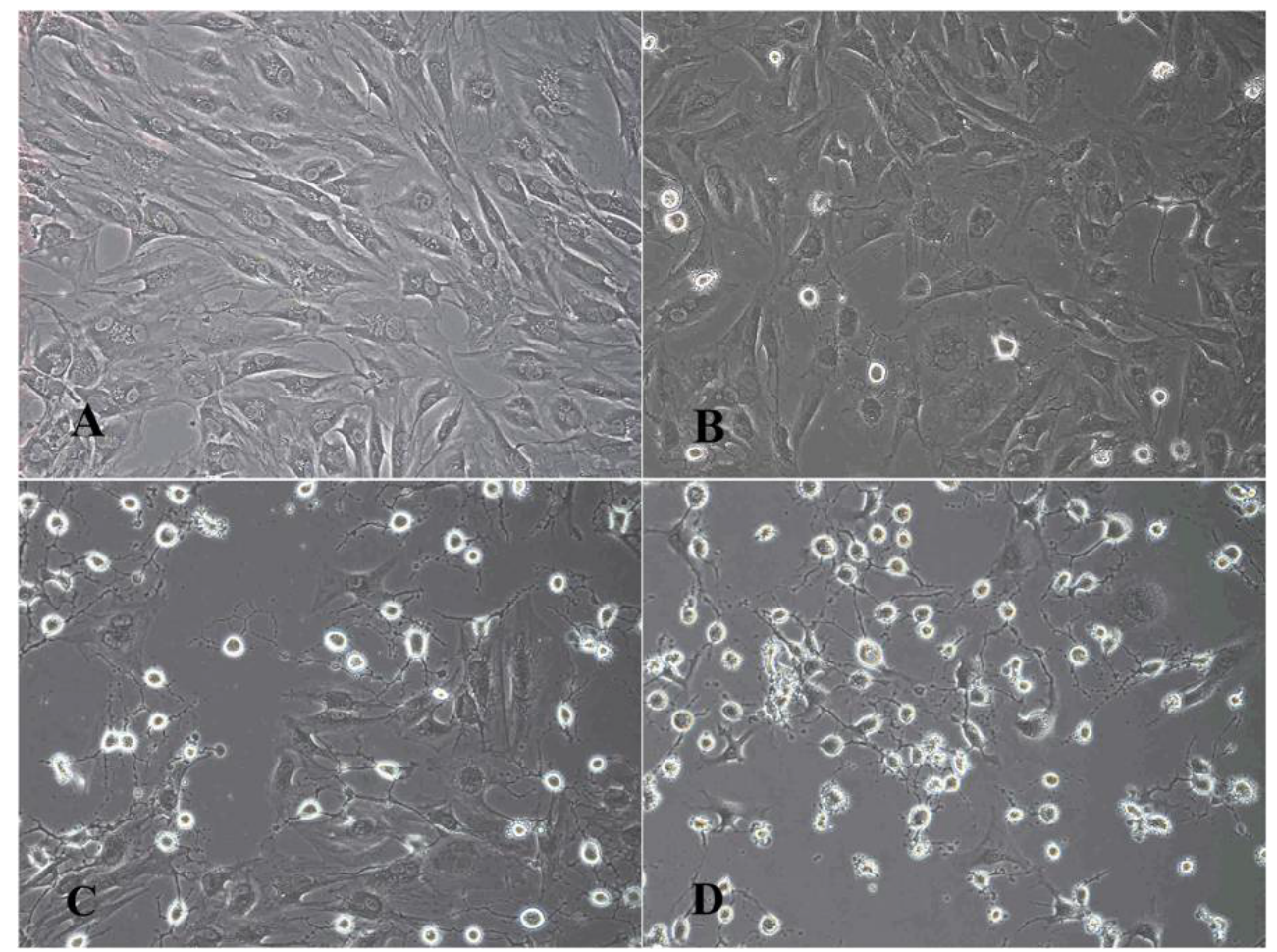

Fig.3: Effect of PM on cellular morphology of AA FLS (24 h)

Cell morphology under microscope (200×) A: Control; B: PM 0.75 $\mu$ M; C: PM 1.5 $\mu$ M group; D: PM $3 \mu M$ 

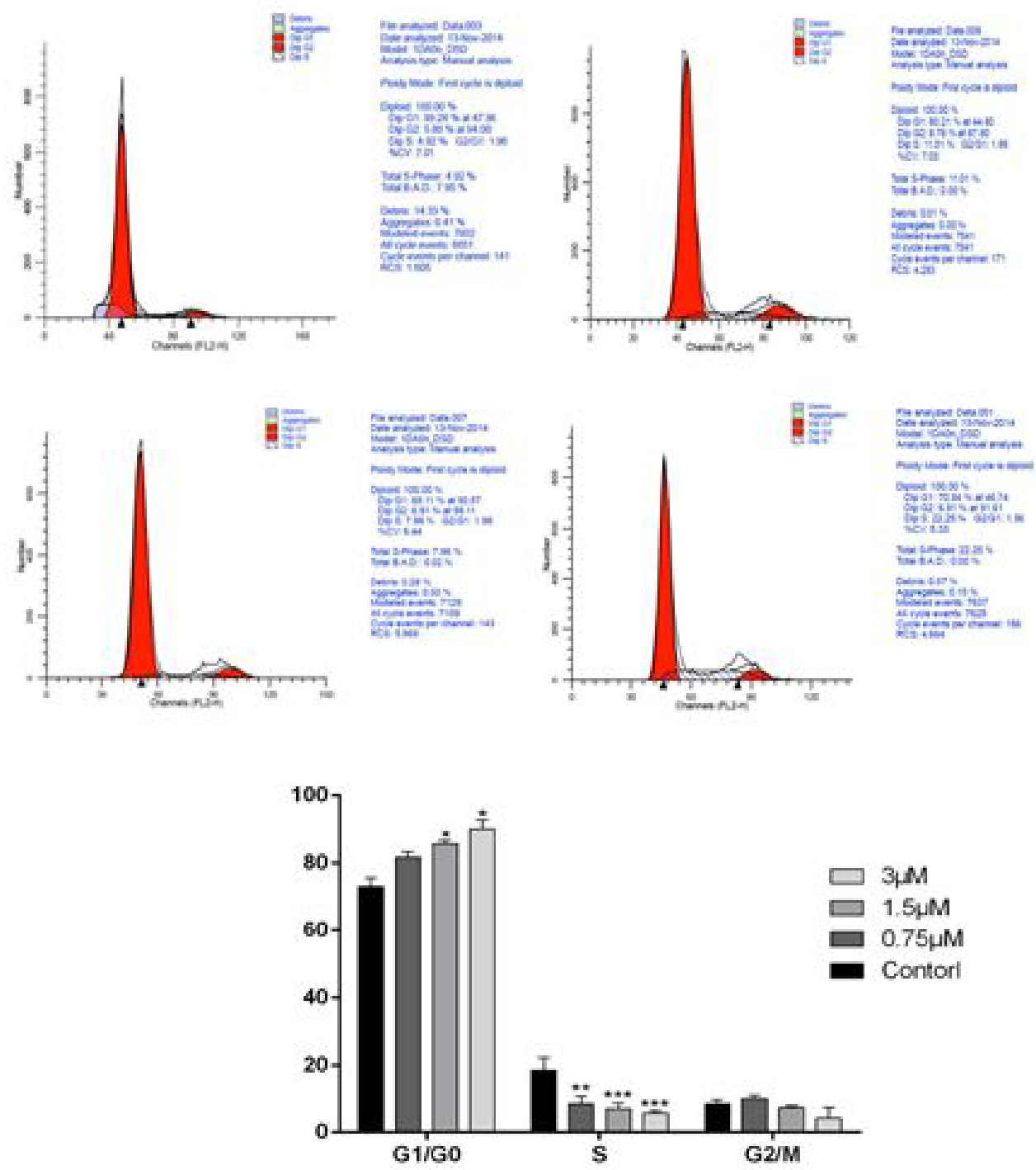

Fig. 4: Effect of PM on cell cycle AA-FLS were treated with either PM (0.75, 1.5 or $3 \mu \mathrm{M})$ for $24 \mathrm{~h}$ ${ }^{*} \mathbf{p}<0.05$ vs. control group, ${ }^{* *} \mathbf{p}<\mathbf{0 . 0 1}$ vs. control group

apoptotic cells), and the control group cells had early apoptosis and the rate is $7.450 \pm 0.723 \%$. The rate of early apoptotic cells of AA FLS in lentilin $0.75 \mu \mathrm{M}$ group was $17.307 \pm 2.519 \%$, the rate of apoptosis of AA FLS cells in $1.5 \mu \mathrm{M}$ group was $47.413 \pm 2.503 \%$, and the rate of apoptosis of AA FLS in $3 \mu \mathrm{M}$ group was $70.970 \pm 4.205 \%$. Compared with the negative control group, there were statistical differences (see fig. 5 for the results).

JC-1 staining results see fig. 6A. After $12 \mathrm{~h}$ of PM $(3 \mu \mathrm{M}, 1.5 \mu \mathrm{M}, 0.75 \mu \mathrm{M})$ intervention, compared with the control group, the proportion of mitochondrial membrane potential depolarized cells in different dose groups of PM increased with the increase in drug dose. The mitochondrial depolarization ratio of the control group was $37.680 \pm 7.244 \%$, and the proportion of AA FLS depolarized cells in the PM $0.75 \mu \mathrm{M}$ group was $38.480 \pm 7.447 \%$, which was not statistically different from the control group. The proportion of AA FLS depolarized cells in the PM group of $1.5 \mu \mathrm{M}$ and $3 \mu \mathrm{M}$ was $54.550 \pm 3.056 \%$ and $91.373 \pm 3.163 \%$, respectively, which was significantly different from the control group. The experimental results suggested that PM can induce AA FLS mitochondrial membrane potential depolarization and is dose-dependent.

Western blot results showed that the relative expression of Bcl-2 in AA FLS cells $(0.855 \pm 0.064)$ was statistically different from normal FLS cells $(0.506 \pm 0.059)$. After $24 \mathrm{~h}$ of $\mathrm{PM}$ intervention, $1.5 \mu \mathrm{M}(0.729 \pm 0.083)$ and $3 \mu \mathrm{M}(0.507 \pm 0.047)$ of PM could down-regulate the relative expression of Bcl-2 in AA FLS cells; comparing the expression of Bax protein in each group, It was found that the relative expression of Bax decreased in AA FLS $(0.529 \pm 0.079)$ and FLS $(0.659 \pm 0.087)$ cells, 


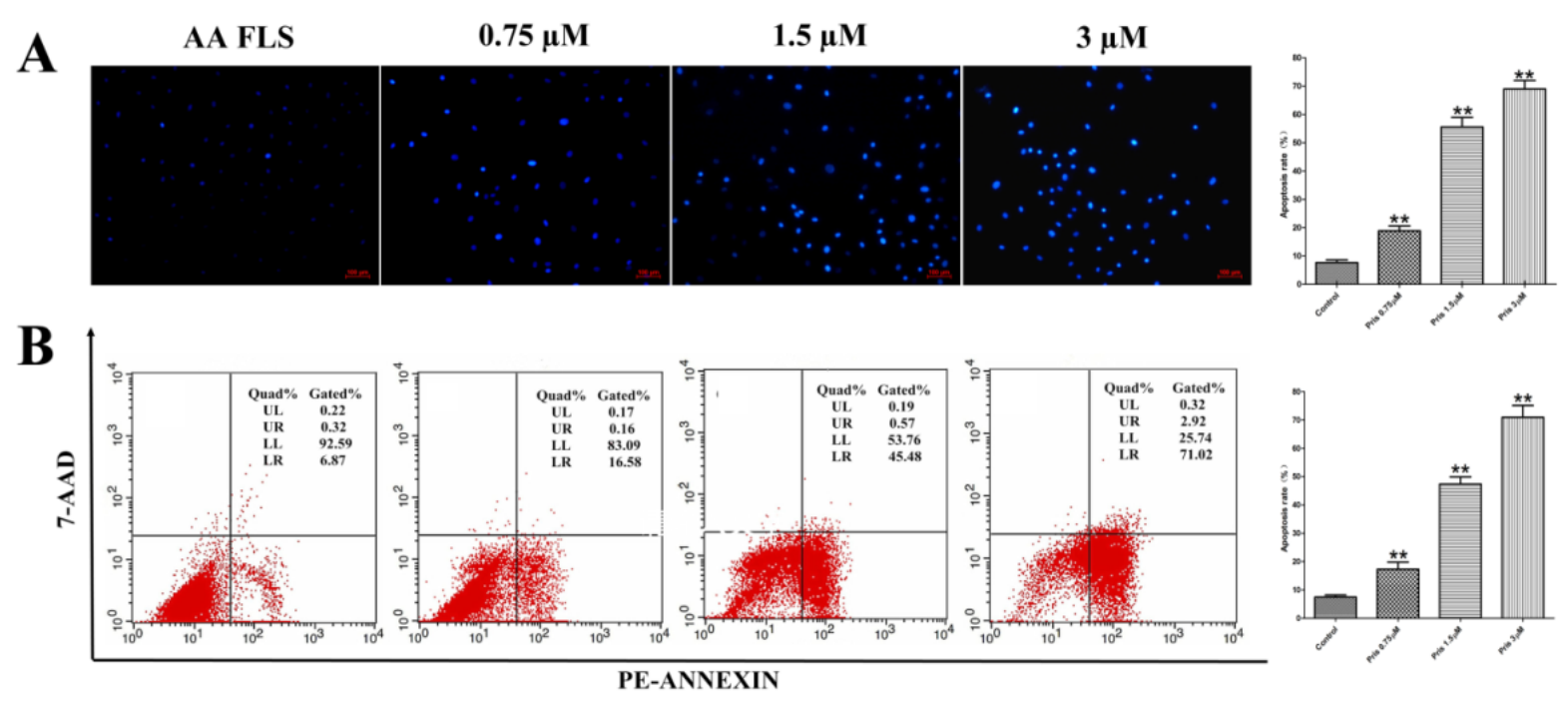

Fig. 5: PM introduce cell apoptosis of AA FLS

A: Hoechst 33258 staining method to detect cell apoptotic ratio of PM on AA FLS apoptosis; B: Flow cytometry detection to detect cell apoptotic ratio of PM on AA FLS

but there was no statistical difference. After $1.5 \mu \mathrm{M}$ and $3 \mu \mathrm{M}$ PM intervention for $24 \mathrm{~h}$, the relative expression of Bax in AA FLS cells showed an increasing trend, which was statistically different from that of AA FLS group (fig. 6B). Compared with FLS cells, the expression of Pro-Caspase-3 protein in AA FLS cells was higher than that in the normal FLS group, but there was no statistical difference. After $24 \mathrm{~h}$ of $1.5 \mu \mathrm{M}$ and $3 \mu \mathrm{M}$ Benglitin treatment, the relative expression of Pro-Caspase-3 protein decreased significantly and was dose-dependent (fig. 6C). Comparing the expression of Cleave-Caspase-3 protein between groups, it was found that Cleave-Caspase-3 protein was not expressed in normal FLS cells. However, the expression of Cleave-Caspase-3 in AA FLS cells was up-regulated and statistically different compared with the FLS group. After $24 \mathrm{~h}$ of $1.5 \mu \mathrm{M}$ and $3 \mu \mathrm{M}$ PM treatment, the relative expression of Cleave-Caspase-3 protein in AA FLS cells was significantly increased, this was statistically different from the AA FLS control group. The experimental results suggest that PM can up-regulate the expression of pro-apoptotic protein Bax in AA FLS cells, and at the same time down-regulate the expression of apoptotic protein Bcl-2 further induce the cleavage of Pro-Caspase- 3 protein and increase the expression of active Cleave-Caspase- 3 protein, resulting in the apoptosis of AA FLS cells.

The exact pathogenesis of RA has not been fully explained so far. Drugs to delay the progression of disease are still the main approach of clinical treatment of RA. Biological agents represented by tumour necrosis factor antagonists and disease retardation represented by methotrexate Anti-rheumatic drugs have shown good clinical efficacy in RA treatment, bringing hope to patients. Due to the high cost of treatment, strong side effects, and long-term infection, the above drugs still cannot fully reach the clinical needs. Therefore, the development of new and effective anti-RA drugs has become the focus and difficulty of anti-RA drug research. A high content of active triterpene geniposide was found from the ethyl acetate fraction of the ethanol extract of maple leaf, which is proved important for anti-RA in this study, and PM was used to verify the significant inhibitory effects on joint slip membrane inflammation and proliferation of AA model rats through experiments. It can reduce the content of Interleukin-6 (IL-6) and IL-17A in synovial tissue of AA rats, inhibit the proliferation of synovial tissue lining layer, reduce the infiltration of inflammatory cells to synovial tissue, and prevent synovial tissue hyperplasia due to joint space occupation, thereby maintaining normal joint cavity structure. It has potential for development of new anti-RA drugs, but PM's pharmacological action mechanism to achieve the above pharmacological effects remains to be experimentally revealed.

In order to clarify the pharmacological mechanism of PM's anti-AA synovial hyperplasia, this study took the primary culture of AA FLS cells in vitro as the research object. First, the difference between AA FLS cells and normal rat FLS cells was compared. The proliferation ability is enhanced, with RA-like hyper proliferative properties. Subsequently, the effect of PM on the 

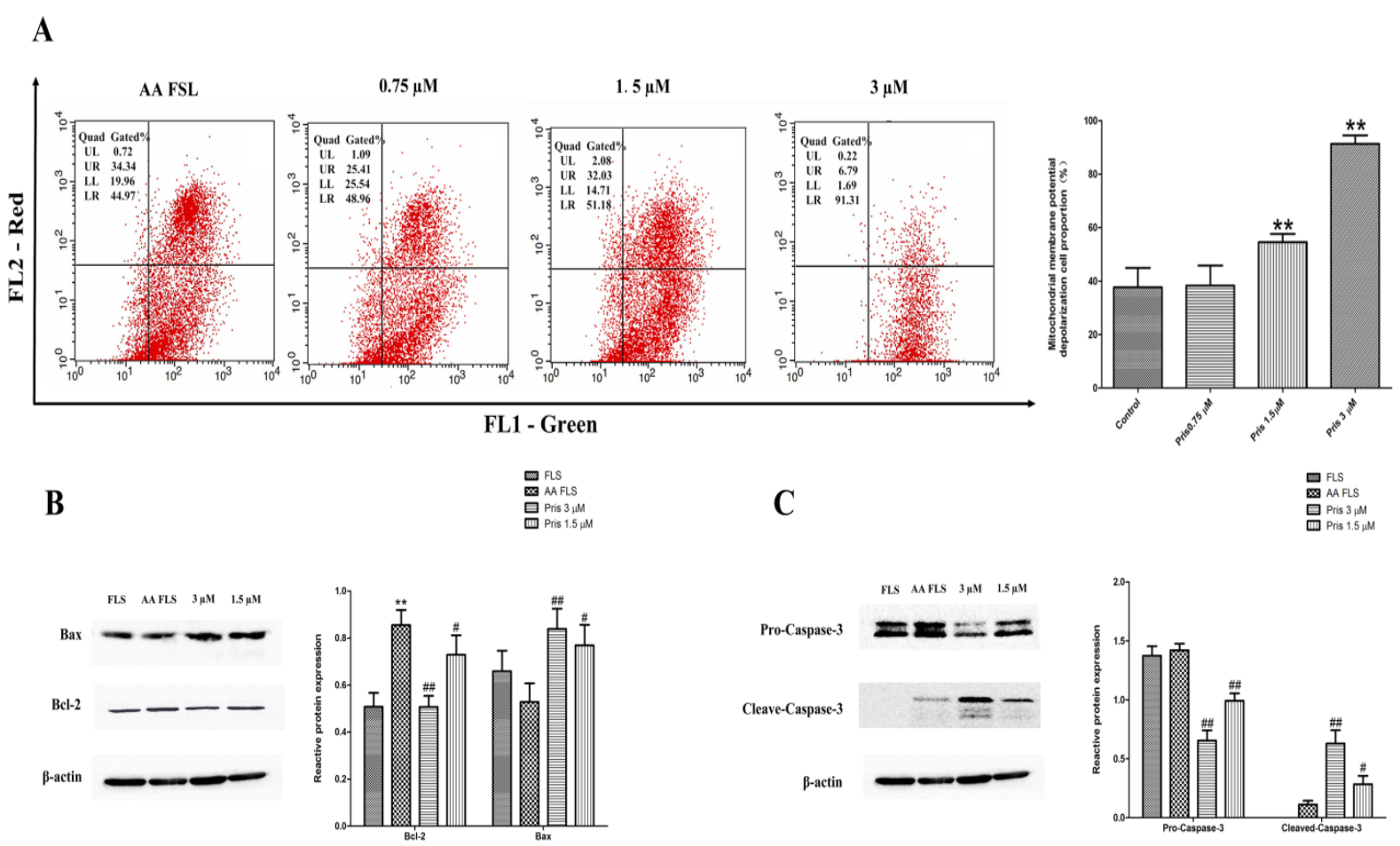

Fig.6: Mitochondrial membrane potential and pathway regulation protein expression.

A: JC-1 detects the effect of genistein on AA FLS mitochondrial membrane potential; B: Bax and Bcl-2 expression; C: ProCaspase- 3 and Cleave-Caspase- 3 expression

proliferation ability of AA FLS cells was analyzed by MTT experiments. The experimental results confirmed that PM could inhibit the proliferation of AA FLS cells in a dose-dependent manner, and proved that PM could inhibit the excessive proliferation of AA FLS cells.

In order to analyse the role of PM in inhibiting the proliferation of AA FLS, firstly we observed the effect of PM on the AA FLS cell cycle. The experimental results confirmed that PM can block the AA FLS cell cycle in the G0/G1 phase, thereby blocking the AA FLS cell proliferation process. Secondly, the anti-apoptotic properties of AA FLS cells compared to normal FLS cells is another important reason for their excessive proliferation. The results of this experiment found that compared with AA FLS and FLS, the expression of important regulatory proteins of the mitochondrial apoptotic pathway, Bax and Bcl-2, was abnormal, in which the expression of Bax protein was decreased, while the expression of anti-apoptotic protein Bcl2 was significantly increased. It suggests that AA FLS cells have certain apoptotic properties. Hoechst 33258 experiment and flow cytometry experiment results confirmed that $\mathrm{PM}$ has the ability to induce AA FLS cell apoptosis. The experimental results confirmed that PM can induce AA FLS cell apoptosis in a concentration range of $0.75 \mu \mathrm{M}$ to $3 \mu \mathrm{M}$ and has a dose-dependent effect. Subsequent cell mitochondrial membrane potential assays confirmed that PM has an effect on AA FLS cell membrane potential, can induce depolarization of mitochondrial membrane potential of AA FLS cells and is dose-dependent. The above results suggest that the effect of PM on the mitochondrial apoptotic pathway is the key drug mechanism for inducing AA FLS. In order to prove the effect of PM on the mitochondrial pathway, this study analyzed the expression of Bax and Bcl-2, important regulatory proteins of the mitochondrial apoptosis pathway, by Western Blotting analysis. The role of maintaining the permeability of the mitochondrial membrane was validated by the analysis. The experimental results confirmed that the typical anti-apoptotic features of Bcl-2 high expression and low Bax expression exist in AA FLS cells, and PM can up-regulate the relative expression of Bax protein in AA FLS cells, while reducing the expression level of anti-apoptotic protein Bcl-2, thereby regulating the anti-apoptotic properties of AA FLS cells. Further observing the effect of PM on the expression of apoptosis initiating protein Caspase-3, the experimental results found that $1.5 \mu \mathrm{M}$ and $3 \mu \mathrm{M}$ PM can promote the inactivation of Pro-Caspase-3 in AA FLS cells and increase the expression of pro- 
apoptotic active Cleave-Caspase-3 protein, thereby promoting the apoptosis of AA FLS cells.

Based on the above, the results of this study confirm that PM's inhibition of synovial tissue proliferation in AA rats is closely related to inhibition of AA FLS proliferation. PM inhibits mitotic proliferation of cells by arresting AA FLS cell cycle in the G0/G1 phase, and by regulating the mitochondrial apoptotic pathway of AA FLS cells to initialize the apoptotic pathway to inhibit the excessive proliferation of AA FLS, and thereby reduce the proliferation of synovial tissue of AA rats, and achieve anti-RA efficacy.

\section{Acknowledgement:}

We thank the Chongqing Science and Technology Bureau for Basic Research and Frontier Exploratory Key Project (cstc2015jcyjBX0082) and Chengdu University of Traditional Chinese Medicine "Xinglin Scholar" Subject Talent Scientific Research Promotion Program Hospital Project (YYZX2019062) for funding this research.

\section{Conflict of interest:}

All authors report no conflicts of interest in this work.

\section{REFERENCES}

1. Falconer J, Murphy AN, Young SP, Clark AR, Tiziani S, Guma $\mathrm{M}$, et al. Synovial cell metabolism and chronic inflammation in rheumatoid arthritis. Arthritis Rheum 2018;70(7):984-99.

2. Leech MT, Morand EF. Fibroblasts and synovial immunity. Curr Opin Pharmacol 2013;13(4):565-9.
3. Bottini N, Firestein GS. Duality of fibroblast-like synoviocytes in RA: passive responders and imprinted aggressors. Nat Rev Rheumatol 2013;9(1):24.

4. Tolboom TC, Zhang YH, Henriquez NK, Nelissen RG, Toes RE, Noteborn MH, et al. Fibroblast-like synoviocytes from patients with rheumatoid arthritis are more sensitive to apoptosis induced by the viral protein, apoptin, than fibroblastlike synoviocytes from trauma patients. Clin Exp Rheumatol 2006;24(2):142-7.

5. Giménez-Cassina A, Danial NN. Regulation of mitochondrial nutrient and energy metabolism by BCL-2 family proteins. Trends Endocrinol Metab 2015;26(4):165-75.

6. Kammouni W, Wong K, Ma G, Firestein GS, Gibson SB, El-Gabalawy HS. Regulation of apoptosis in fibroblast-like synoviocytes by the hypoxia-induced Bcl-2 family member Bcl-2/adenovirus E1B 19-kd protein-interacting protein 3 . Arthritis Rheum 2007;56(9):2854-63.

7. Byun HS, Song JK, Kim YR, Piao L, Won M, Park KA, et al. Caspase- 8 has an essential role in resveratrol-induced apoptosis of rheumatoid fibroblast-like synoviocytes. Rheumatology 2008;47(3):301-8.

8. Tang WH, Bai ST, Tong L, Duan WJ, Su JW, Chen JX, et al. Chemical constituents from Celastrus aculeatus Merr. Biochem Syst Ecol 2014;54:78-82.

9. 9. Tong L, Nanjundaiah SM, Venkatesha SH, Astry B, Yu H, Moudgil KD. Pristimerin, a naturally occurring triterpenoid, protects against autoimmune arthritis by modulating the cellular and soluble immune mediators of inflammation and tissue damage. Clin Immunol 2014;155(2):220-30.

10. 10. Tong L, Moudgil KD. Celastrus aculeatusMerr. Suppresses the induction and progression of autoimmune arthritis by modulating immune response to heat-shock protein 65 . Arthritis Res Ther 2007;9(4):R70.

11. 11. Gao W, Deng Q, Bai S, Tong L. Establishment and characteristic analysis of fibroblast-like synoviocytes in rats with adjuvant arthritis. Chinese Pharmacol Bull 2015;1(12):1693-8. 